\title{
Particles, Holes, and Solitons: A Matrix Product State Approach
}

\author{
Damian Draxler, ${ }^{1}$ Jutho Haegeman, ${ }^{2}$ Tobias J. Osborne, ${ }^{3}$ Vid Stojevic, ${ }^{1,2}$ Laurens Vanderstraeten, ${ }^{2}$ and Frank Verstraete ${ }^{1,2}$ \\ ${ }^{1}$ Vienna Center for Quantum Science, Universität Wien, Boltzmanngasse 5, A-1090 Wien, Austria \\ ${ }^{2}$ Department of Physics and Astronomy, Ghent University, Krijgslaan 281- S9, B-9000 Ghent, Belgium \\ ${ }^{3}$ Institute of Theoretical Physics and Riemann Center for Geometry and Physics, Leibniz Universität Hannover,
}

Appelstrasse 2, D-30167 Hannover, Germany

(Received 20 December 2012; published 9 July 2013)

\begin{abstract}
We introduce a variational method for calculating dispersion relations of translation invariant $(1+1)$ dimensional quantum field theories. The method is based on continuous matrix product states and can be implemented efficiently. We study the critical Lieb-Liniger model as a benchmark and excellent agreement with the exact solution is found. Additionally, we observe solitonic signatures of Lieb's type II excitation. In addition, a nonintegrable model is introduced where a $U(1)$-symmetry breaking term is added to the Lieb-Liniger Hamiltonian. For this model we find evidence of a nontrivial bound-state excitation in the dispersion relation.
\end{abstract}

DOI: 10.1103/PhysRevLett.111.020402

PACS numbers: 05.30.- d, 03.67.Ac, 03.75.Lm, 75.40.Mg

The past decades have witnessed an explosion in the experimental realization of strongly correlated onedimensional quantum systems [1]. Often a discretized description in terms of a lattice Hamiltonian is possible, which can then be studied using White's density matrix renormalization group [2]. The underlying variational ansatz, the set of matrix product states (MPS) [3-7], explains the success of this method and has been used to develop generalizations beyond the setting of ground states, e.g., to the study of time evolution [8,9], dissipative dynamics [10,11], and dispersion relations $[12,13]$.

The use of elongated optical or magnetic atom traps has opened the possibility of creating one-dimensional quantum gases in the lab [14-19]. It is natural to study these systems directly using quantum fields, without resorting to a lattice discretization. A continuum limit of the class of matrix product states, known as continuous matrix product states (cMPS) [20-22], was recently developed and has demonstrated its ability to provide an efficient description of the ground-state properties of the Lieb-Liniger (LL) model [23].

Apart from ground-state properties, there has also been experimental interest in localized excitations in these systems [24-27]. While Lieb determined the spectrum of excitations for the LL model [28], a systematic method for studying excitations of nonintegrable quantum fields is still lacking. In this Letter we fill the gap by extending the recently introduced ansatz for excitations of translation invariant spin chains in the thermodynamic limit [13] to the setting of cMPS. This yields a new variational ansatz for elementary excitations of translation invariant quantum fields that allows us to simulate dispersion relations for integrable and nonintegrable models alike. The corresponding variational states are faithful eigenstates and have therefore an infinite lifetime, out of which we can construct localized wave packets by taking linear combinations. With this method, we can reconstruct the spectrum of the LL Hamiltonian and illustrate the solitonic effects in Lieb's type II excitation-first observed in Refs. $[29,30]$ in the weak-interaction limit-for arbitrary interaction strength. We can equally well construct the spectrum for nonintegrable models, which we illustrate by adding a pairing term to the LL Hamiltonian which opens a gap. For a certain parameter regime, our method provides strong evidence for the existence of a nontrivial bound state.

A cMPS for a translation invariant infinite system with open boundary conditions is defined as [20]

$$
|\Psi(Q, R)\rangle=v_{L}^{\dagger}\left(\mathcal{P} e \int_{-\infty}^{\infty} d x\left[Q \otimes \mathbb{1}+R \otimes \hat{\psi}^{\dagger}(x)\right]\right) v_{R}|\Omega\rangle,
$$

where $Q, R \in \mathbb{C}^{D \times D}, v_{L}$ and $v_{R}$ are $D$-dimensional boundary vectors acting on an ancillary system, $|\Omega\rangle$ is the Fock vacuum, and $\mathcal{P}$ the path-ordering operator. For bosonic systems the field operators satisfy the commutation relation $\left[\hat{\psi}(x), \hat{\psi}^{\dagger}(y)\right]=\delta(x-y)$. For a generic normalizable cMPS, all eigenvalues of the transfer matrix $T=Q \otimes \mathbb{1}+\mathbb{1} \otimes \bar{Q}+R \otimes \bar{R}$ have nonpositive real part and there is a nondegenerate zero eigenvalue. The corresponding left and right zero eigenvectors $\langle l| T=$ $0, T|r\rangle=0$ may be reshaped to give positive, Hermitian matrices $l$ and $r$ which have full rank and are normalized so that $\langle l \mid r\rangle=\operatorname{Tr}(l r)=1$. Since the boundary vectors have no variational importance, they are chosen so that the state has norm 1, i.e., $\langle\Psi(\bar{Q}, \bar{R}) \mid \Psi(Q, R)\rangle=$ $\left(v_{L}^{\dagger} \otimes v_{L}^{\top}\right)|r\rangle\langle l|\left(v_{R} \otimes \bar{v}_{R}\right)=\left(v_{L}^{\dagger} r v_{L}\right)\left(v_{R}^{\dagger} l v_{R}\right)=1$.

Suppose we have approximated the ground state of a system as a cMPS parametrized by the matrices $(Q, R)$. An ansatz for a particlelike eigenstate or excitation is obtained by locally replacing the matrices $Q$ and $R$ by $V$ and $W-$ which has the effect of perturbing the ground state in a spatial region of the size of the correlation length-and 
then building a state with definite momentum via a plane wave superposition:

$$
\begin{aligned}
\left|\Phi_{p}(V, W)\right\rangle:= & \int_{-\infty}^{\infty} d x e^{i p x} v_{L}^{\dagger} \hat{U}_{1}(-\infty, x) \\
& \times\left[V \otimes \mathbb{1}+W \otimes \hat{\psi}^{\dagger}(x)\right] \hat{U}_{2}(x, \infty) v_{R}|\Omega\rangle,
\end{aligned}
$$

where $\quad \hat{U}_{a}(x, y)=\mathcal{P} \exp \left\{\int_{x}^{y} d z\left[Q_{a} \otimes \mathbb{1}+R_{a} \otimes \hat{\psi}^{\dagger}(z)\right]\right\}$ for $a=1,2$. The crucial feature here is a single strictly local disturbance described by the matrices $V, W \in \mathbb{C}^{D \times D}$, which is nevertheless able to influence the state up to a distance determined by the bond dimension and appears to efficiently capture single-particle excitations, as we illustrate below. The states $\left|\Phi_{p}(V, W)\right\rangle$ are momentum eigenstates and obey a $\delta$ orthogonality. Note that they depend linearly on the variational parameters $V$ and $W$, so they span a linear subspace of Hilbert space. Asymptotically the states $\left|\Phi_{p}(V, W)\right\rangle$ look like $\left|\Psi\left(Q_{1}, R_{1}\right)\right\rangle$ at $x=-\infty$ and $\left|\Psi\left(Q_{2}, R_{2}\right)\right\rangle$ at $x=+\infty$, which are supposed to be equally good but potentially different ground states (in the case of symmetry breaking). Our ansatz thus includes the possibility of capturing topologically nontrivial excitations, henceforth referred to as topological excitations for the sake of brevity. For $\left|\Psi\left(Q_{1}, R_{1}\right)\right\rangle=\left|\Psi\left(Q_{2}, R_{2}\right)\right\rangle$, the ansatz describes (topologically) trivial excitations and can be interpreted as a state in the momentum sector $p$ of the tangent space obtained by an infinitesimal position dependent variation of $|\Psi(Q, R)\rangle$ [22].

To compute excitations we apply the Rayleigh-Ritz method, which results in a generalized eigenvalue problem for an effective Hamiltonian. In our case the generalized eigenvalue equation is given by

$$
H_{p}\left[\begin{array}{c}
V \\
W
\end{array}\right]=E N_{p}\left[\begin{array}{c}
V \\
W
\end{array}\right],
$$

with $\left[\begin{array}{l}V \\ W\end{array}\right]$ being the $2 D^{2}$-dimensional vector corresponding to $V$ and $W, E$ is the energy, $H_{p}$ the effective Hamiltonian, and $N_{p}$ the effective norm matrix. Both $H_{p}$ and $N_{p}$ are $\left(2 D^{2} \times 2 D^{2}\right)$-dimensional matrices defined by

$$
\begin{aligned}
\left\langle\Phi_{p}(\bar{V}, \bar{W})\left|\hat{H}-E_{0}\right| \Phi_{p^{\prime}}(V, W)\right\rangle= & 2 \pi \delta\left(p-p^{\prime}\right)\left[V^{\dagger} W^{\dagger}\right] \\
& \times H_{p}\left[\begin{array}{c}
V \\
W
\end{array}\right], \\
\left\langle\Phi_{p}(\bar{V}, \bar{W}) \mid \Phi_{p^{\prime}}(V, W)\right\rangle= & 2 \pi \delta\left(p-p^{\prime}\right)\left[V^{\dagger} W^{\dagger}\right] \\
& \times N_{p}\left[\begin{array}{c}
V \\
W
\end{array}\right],
\end{aligned}
$$

where $E_{0}$ is the ground-state energy obtained with the ground-state approximation $|\Psi(Q, R)\rangle$.

Note that $H_{p}$ and $N_{p}$ have zero eigenvalues corresponding to a redundancy in the representation of the states $\left|\Phi_{p}(V, W)\right\rangle$, which can be traced back to the gauge invariance of the states $|\Psi(Q, R)\rangle$ under the transformation $Q \leftarrow g^{-1} Q g$ and $R \leftarrow g^{-1} R g$ [22]. One finds that for all
$X \in \mathbb{C}^{D \times D}, \quad\left|\Phi_{p}([X, Q]+i p X,[X, R])\right\rangle=0 . \quad$ Hence, $\left|\Phi_{p}\left(V^{\prime}, W^{\prime}\right)\right\rangle=\left|\Phi_{p}(V, W)\right\rangle$ if $V^{\prime}=V+[X, Q]+i p X$ and $W^{\prime}=W+[X, R]$. If, for $p=0$, one also restricts to states orthogonal to the ground state by imposing $\langle l|(V \otimes$ $\mathbb{1}+W \otimes \bar{R})|r\rangle=0$, there are $D^{2}$ redundant degrees of freedom for every momentum $p$. These can be eliminated by constraining $V$ and $W$ to satisfy a "gauge-fixing" condition such as

$$
\langle l|(V \otimes \mathbb{1}+W \otimes \bar{R})=0,
$$

with $\langle l|$ the left eigenvector of $T_{11}$, the transfer matrix corresponding to $\left|\Psi\left(Q_{1}, R_{1}\right)\right\rangle$. It can be shown that this choice of gauge reduces the effective norm matrix to the identity (Ref. [22] and Supplemental Material [31]), so that the Rayleigh-Ritz problem becomes an ordinary eigenvalue problem. The explicit calculation of the effective Hamiltonian $H_{p}$ in this gauge is more involved and is derived in full detail in the Supplemental Material [31]. The lowest eigenvalues of $H_{p}$ can then be obtained with a computational time scaling as $\mathcal{O}\left(D^{3}\right)$ using a sparse eigensolver exploiting the tensor product structure of the effective Hamiltonian.

We now discuss the results obtained for the LL model. The LL Hamiltonian is given by

$$
\hat{H}_{\mathrm{LL}}=\int_{-\infty}^{+\infty}\left[\frac{d \hat{\psi}^{\dagger}}{d x} \frac{d \hat{\psi}}{d x}-\mu \hat{\psi}^{\dagger} \hat{\psi}+c \hat{\psi}^{\dagger} \hat{\psi}^{\dagger} \hat{\psi} \hat{\psi}\right] d x,
$$

with a repulsive interaction strength $c>0$ and where the argument of the field operators has been omitted for the sake of brevity. Since a variational approach targets the lowest energy state, this Hamiltonian was formulated in the grand-canonical ensemble (with chemical potential $\mu>0$ ). The Hamiltonian is gapless and only depends on a single parameter $\gamma=c / \rho$, with $\rho$ the ground-state particle density which is set by $\mu$. The cMPS ansatz generalizes a coherent state ansatz and breaks the $U(1)$ symmetry of the model, whereas the exact Bethe-ansatz ground state does not and has a fixed total number of particles. This follows from the fact that it is often energetically beneficial to break the symmetry in the presence of a constraint on the total amount of entanglement. Hence, the order parameter $\langle\Psi(\bar{Q}, \bar{R})|\hat{\psi}| \Psi(Q, R)\rangle \neq 0$ (and in fact slowly converges to 0 for increasing $D)$. For any $\theta \in[0,2 \pi),\left|\Psi\left(Q, e^{i \theta} R\right)\right\rangle$ is again a valid ground state so that we can also consider topological excitations interpolating between two different ground states characterized by a different order parameter. They can also be understood as momentum superpositions of a local perturbation at position $x$ which has a halfinfinite string $\hat{S}(x)=\exp \left[i \theta \int_{-\infty}^{x} \hat{\psi}^{\dagger}(z) \hat{\psi}(z) d z\right]$ attached to it. Even for the exact solution with fixed particle number $N$, the elementary particle $(N+1)$ and hole $(N-1)$ excitations have a topological nature and need to be studied using antiperiodic boundary conditions [32]. For $\theta=\pi$, 
the string $\hat{S}(x)$ has exactly the effect of flipping the sign of the field operators at $-\infty\left[\hat{S}(x)^{\dagger} \hat{\psi}(-\infty) \hat{S}(x)=-\hat{\psi}(-\infty)\right]$, and Fig. 1 does indeed confirm that the elementary excitations are perfectly captured by using the ansatz for topological excitations with the choice $Q_{1}=Q_{2}=Q$, $R_{1}=-R_{2}=R$. The dispersion relation is centered around 0 and the hole branch (red stars) is obtained as the lowest excitation energy with momentum between $-\pi \rho$ and $+\pi \rho$, with $\rho$ the particle density. The particle branch (blue diamonds) shows the eigenvalues of the eigenvectors that have the largest overlap with the state $\int_{-\infty}^{\infty} d x e^{i p x} \hat{\psi}^{\dagger}(x) e^{i \pi \int_{-\infty}^{x} \hat{\psi}^{\dagger}(z) \hat{\psi}(z) d z}|\Psi(Q, R)\rangle$. All parameters plotted in the figures are normalized such that they are dimensionless $\left(p / \rho, e / \rho^{2}\right)$, as in Ref. [23].

Lieb determined the spectrum with fixed particle number (i.e., topologically trivial) in first quantization [28] and isolated two types of excitations, which he labeled type I and type II excitations. Either can be used to construct the full spectrum of excitations with equal particle number. Figure 2 shows the eigenvalues of the effective Hamiltonian $H_{p}$ as a function of the momentum $p$ obtained using the trivial ansatz $\left(Q_{1}=Q_{2}\right.$ and $\left.R_{1}=R_{2}\right)$. However, it is well known [32] that the type I excitations should be understood as one hole at momentum $-\pi \rho$ plus one particle with momentum $p \geq \pi \rho$, whereas the type II excitations at momentum $p$ are obtained by combining one particle with momentum $\pi \rho$ plus one hole with momentum $-\pi \rho \leq p \leq \pi \rho$. By combining momentum and variational energies of our topological excitations according to this recipe, we can accurately reproduce (5 digits of precision) the Bethe-ansatz dispersion relations of Lieb's type I [blue diamonds in Figs. 2 and 1(b)] and type II

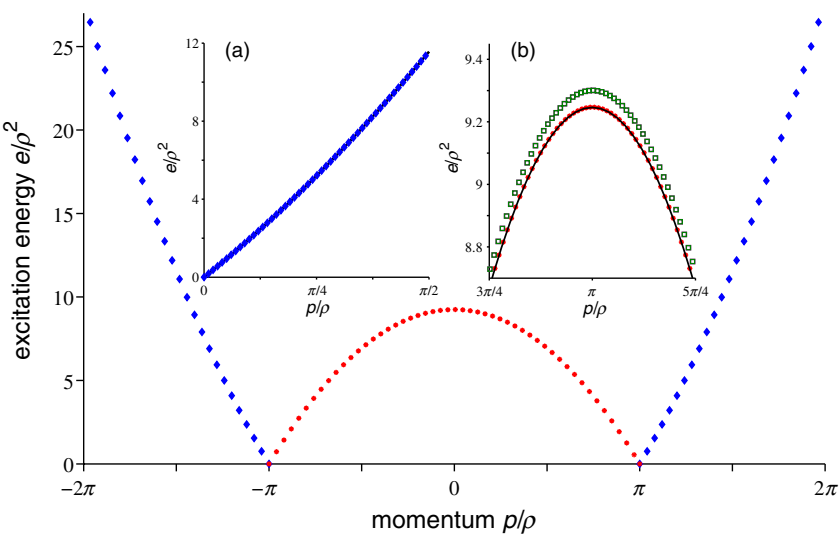

FIG. 1 (color online). Elementary topological excitation branches for $\gamma=c / \rho \approx 60.16$ at $D=64$ with $\theta=\pi$. Blue diamonds are particle excitations and red stars hole excitations. (a) Dispersion relation of Lieb's type I excitation obtained from the topological ansatz (blue diamonds) and from the Betheansatz solution (black line). (b) Dispersion relation of Lieb's type II excitation obtained from the topological ansatz (red stars), the trivial ansatz (green squares), and from the Betheansatz solution (black line). excitations [red stars in Figs. 2 and 1(b)]. If we would have used the trivial excitation energies directly [also sketched in Fig. 1(b)], a worse precision would have been obtained, as this single particle ansatz cannot accurately represent the two unbound constituents of Lieb's type I and type II excitations and tries to confine them into a small spatial region. Since the repulsive Lieb-Liniger model does not have any bound states [32], all trivial excitations have a similar structure consisting of an even sum of topological hole and particle excitations.

Without knowing the exact result, this information could be inferred from looking at the convergence of the variational energies as a function of increasing $D$. There are two competing effects within our variational strategy. Firstly, by modifying $Q$ and $R$ locally, we assume that the excitation is confined in a spatial region, the width of which is set by the bond dimension. This is the variational approximation and it produces a positive variational error. Secondly, we subtract from the Hamiltonian a variational estimate of the ground-state energy which is too large. This second effect results in a negative error and is dominant for truly elementary excitations for which the assumed locality is valid and the first error is negligible. If an excited state cannot be approximated locally, e.g., an excitation that is composed of several unbound elementary excitations, then the first effect will likely be dominant as the ansatz confines these different excitations in a finite spatial region. Indeed, for increasing $D$, the particle and hole energies in Fig. 1 are increasing (which is true for all $\gamma \gtrsim 3$ ) because a smaller variational estimate for the ground-state energy is subtracted. In contrast, the energies of trivial excitations in Fig. 2 decrease for increasing $D$, in line with our expectations.

Our ansatz does not depend on $\gamma$ and works in principle equally well for both limiting cases $\gamma \rightarrow \infty$ and $\gamma \rightarrow 0$, as is confirmed by the results for small values of $\gamma$ in the Supplemental Material [31]. However, for small $\gamma(\gamma \lesssim 3)$ we observe a transition of the topological hole excitation to

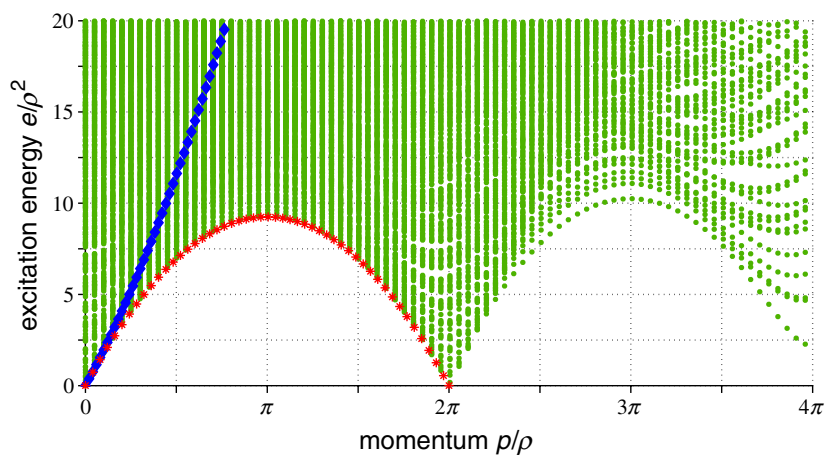

FIG. 2 (color online). Dispersion relation for the Lieb-Liniger model with $D=64$ for $\gamma=c / \rho \approx 60.16$. Bulk excitations (dots) are trivial excitations. Blue diamonds (type I) and red stars (type II) are obtained by combining momenta and energy of two topological excitations (hole and particle) of Fig. 1. 
a solitonic excitation. This observation was first discussed in Refs. [29,30] and recently received renewed attention following the experimental realization [26,33,34]. In the Tonks-Girardeau (TG) limit (large $\gamma$ ) [35], the hole excitation maps exactly to the fermionlike hole excitation and produces a change in particle number of -1 . For very small $\gamma$, the hole excitation is related to the classical dark soliton of the nonlinear Schrödinger equation [36] and produces a large change in particle number [34]. The relative change in particle number can easily be calculated using our ansatz. It is illustrated in Fig. 3 for momentum $p=0$, and the results perfectly coincide with the recent exact calculations [34].

Next, we study a nonintegrable model obtained by adding a pairing term to $\hat{H}_{\mathrm{LL}}$ that breaks the $U(1)$ symmetry down to a residual $\mathbb{Z}_{2}$ symmetry:

$$
\hat{H}^{\prime}=\hat{H}_{\mathrm{LL}}+\int_{-\infty}^{\infty} d x\left(u \hat{\psi}^{\dagger} \hat{\psi}^{\dagger}+\bar{u} \hat{\psi} \hat{\psi}\right) .
$$

As long as $\mu>0$, the cMPS ground-state approximation $|\Psi(Q, R)\rangle$ spontaneously breaks the $\mathbb{Z}_{2}$ symmetry for any nonzero pairing strength $u \in \mathbb{C}_{0}$, and a second ground state $|\Psi(Q,-R)\rangle$ is obtained. This also opens up a gap in the spectrum. The magnitude of the order parameter $\langle\Psi(\bar{Q}, \bar{R})|\hat{\psi}(x)| \Psi(Q, R)\rangle$ is determined by the competing effects of the pairing term and the repulsive interaction. As an example, we consider an intermediate parameter range where we have found strong evidence that the lowest lying excitation in the trivial spectrum is a bound state. In the top plot of Fig. 4, the lowest lying trivial excitations of $\hat{H}^{\prime}$ for $\gamma \approx 26.4, \mu=1$, and $u=1$ with $D=22$ are shown with red circles. Because of the symmetry breaking, we can again construct topological excitations (right-hand inset of Fig. 4), for which the lowest lying eigenvalues constitute an isolated branch that can be interpreted as the kink excitation that interpolates between the two degenerate

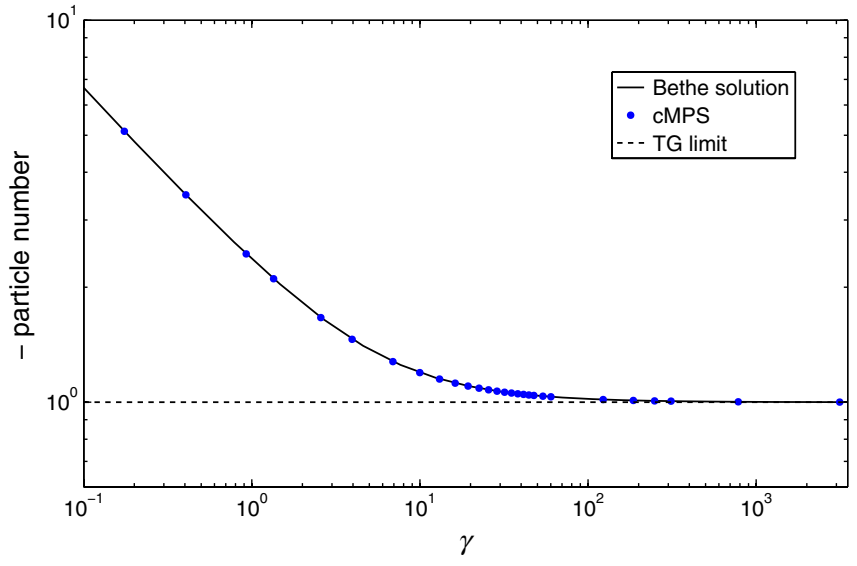

FIG. 3 (color online). The change in particle number expectation value for the hole state at momentum $p=0$ as a function of $\gamma$, as predicted by the Bethe solution [34] and with the cMPS excitation ansatz. ground states. The blue dots in the trivial spectrum of Fig. 4 are obtained by considering all possible pairs of two such topological excitations by adding their momentum and energy. Around momentum zero, the lowest lying trivial excitation produced by the trivial ansatz lies well below the two-kink continuum starting at twice the kink mass.

It is tempting to interpret this excitation as a bound state of two kinks, and this claim is further supported by considering the convergence behavior of (twice) the kink mass and the two lowest trivial excitation energies as a function of $D$ in the bottom plot of Fig. 4. The kink is the elementary excitation; it has a negative error that vanishes quickly for increasing $D$. As in the LL case, unbound multiparticle states are not expected to converge quickly, because the different constituents are confined within a spatial region, the size of which is determined by the bond dimension [37]. For the energy of a two-particle state, we thus expect a positive finite size error. The energy of a bound state should also exhibit a large positive error as long as the
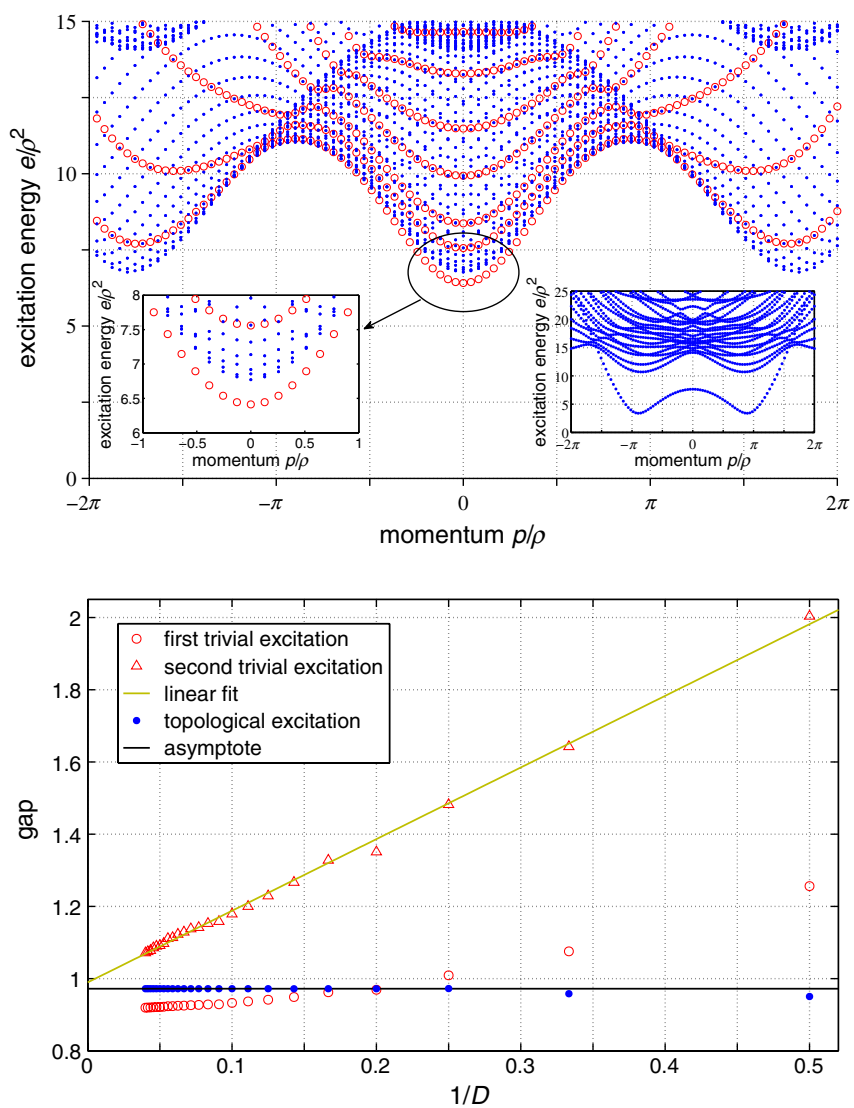

FIG. 4 (color online). Top: Dispersion relation of $\hat{H}^{\prime}$ with $D=22$ for $\gamma \approx 26.4, \mu=1$, and $u=1$. Blue dots are the pairwise sum of all topological excitations. Red circles are trivial excitations and the right-hand inset shows the topological spectrum. Bottom: Convergence of the two lowest trivial eigenvalues and the lowest two-kink excitation energy as a function of $D^{-1}$ at $p=0$. 
spatial support of the excitation ansatz is below the binding length, but should then stagnate when the binding length is exceeded by increasing $D$. Figure 4 confirms that the lowest lying energy of the trivial ansatz converges to a value well below twice the kink mass in a way that is characteristic for a bound state.

In conclusion, we have introduced a variational ansatz for the study of elementary excitations for one-dimensional quantum field theories, based on continuous matrix product states. The ansatz produces very accurate results even for the critical Lieb-Liniger model. The local aspect of the ansatz made the solitonic feature of the lowest lying hole eigenstates of the LL model very explicit, and the particle number in those solitons could be reproduced to great accuracy. We also applied the method to a gapped nonintegrable variant of LL, and obtained strong evidence for the existence of a bound state. As an outlook, we can remark that this method provides a stepping stone to a further understanding of the low-energy dynamics of one-dimensional interacting quantum field theories. In order to calculate, e.g., dynamical correlation functions, it would be interesting to study scattering states of topological excitations [38].

We thank G.E. Astrakharchik and L.P. Pitaevskii for sharing the particle number data from Ref. [34] and J.S. Caux, F. Essler, F. D. M. Haldane, and V. Korepin for stimulating discussions. We acknowledge support from the EU grants QUERG, by the Austrian FWF SFB grants FoQus and ViCoM, and an Odysseus grant from the Research Foundation Flanders. L. V. is supported by a Doctoral Scholarship of the Special Research Fund UGent. T. J. O. is supported by the EU grant QFTCMPS and the cluster of excellence EXC 201 Quantum Engineering and Space-Time Research.

[1] T. Giamarchi, Quantum Physics in One Dimension (Clarendon Press, Oxford, 2003).

[2] S. R. White, Phys. Rev. Lett. 69, 2863 (1992).

[3] I. Affleck, T. Kennedy, E. H. Lieb, and H. Tasaki, Phys. Rev. Lett. 59, 799 (1987).

[4] M. Fannes, B. Nachtergaele, and R. Werner, Commun. Math. Phys. 144, 443 (1992).

[5] S. Östlund and S. Rommer, Phys. Rev. Lett. 75, 3537 (1995).

[6] F. Verstraete, V. Murg, and J. Cirac, Adv. Phys. 57, 143 (2008).

[7] J. I. Cirac and F. Verstraete, J. Phys. A 42, 504004 (2009).

[8] G. Vidal, Phys. Rev. Lett. 93, 040502 (2004).

[9] J. Haegeman, J. I. Cirac, T. J. Osborne, I. Pižorn, H. Verschelde, and F. Verstraete, Phys. Rev. Lett. 107, 070601 (2011).

[10] F. Verstraete, J. J. García-Ripoll, and J. I. Cirac, Phys. Rev. Lett. 93, 207204 (2004).

[11] M. Zwolak and G. Vidal, Phys. Rev. Lett. 93, 207205 (2004).
[12] B. Pirvu, J. Haegeman, and F. Verstraete, Phys. Rev. B 85, 035130 (2012).

[13] J. Haegeman, B. Pirvu, D. J. Weir, J. I. Cirac, T. J. Osborne, H. Verschelde, and F. Verstraete, Phys. Rev. B 85, 100408 (2012).

[14] A. Görlitz, J. M. Vogels, A. E. Leanhardt, C. Raman, T. L. Gustavson, J. R. Abo-Shaeer, A. P. Chikkatur, S. Gupta, S. Inouye, T. Rosenband, and W. Ketterle, Phys. Rev. Lett. 87, 130402 (2001).

[15] H. Moritz, T. Stöferle, M. Köhl, and T. Esslinger, Phys. Rev. Lett. 91, 250402 (2003).

[16] T. Kinoshita, T. Wenger, and D. S. Weiss, Science 305, 1125 (2004).

[17] T. Kinoshita, T. Wenger, and D. S. Weiss, Phys. Rev. Lett. 95, 190406 (2005).

[18] J. Esteve, J.-B. Trebbia, T. Schumm, A. Aspect, C. I. Westbrook, and I. Bouchoule, Phys. Rev. Lett. 96, 130403 (2006).

[19] P. Krüger, S. Hofferberth, I. E. Mazets, I. Lesanovsky, and J. Schmiedmayer, Phys. Rev. Lett. 105, 265302 (2010).

[20] F. Verstraete and J. I. Cirac, Phys. Rev. Lett. 104, 190405 (2010).

[21] T. J. Osborne, J. Eisert, and F. Verstraete, Phys. Rev. Lett. 105, 260401 (2010).

[22] J. Haegeman, J. I. Cirac, T. J. Osborne, and F. Verstraete, arXiv:1211.3935.

[23] E. H. Lieb and W. Liniger, Phys. Rev. 130, 1605 (1963).

[24] S. Burger, K. Bongs, S. Dettmer, W. Ertmer, K. Sengstock, A. Sanpera, G. V. Shlyapnikov, and M. Lewenstein, Phys. Rev. Lett. 83, 5198 (1999).

[25] C. Becker, S. Stellmer, P. Soltan-Panahi, S. Dörscher, M. Baumert, E.-M. Richter, J. Kronjäger, K. Bongs, and K. Sengstock, Nat. Phys. 4, 496 (2008).

[26] S. Stellmer, C. Becker, P. Soltan-Panahi, E.-M. Richter, S. Dörscher, M. Baumert, J. Kronjäger, K. Bongs, and K. Sengstock, Phys. Rev. Lett. 101, 120406 (2008).

[27] A. Weller, J. P. Ronzheimer, C. Gross, J. Esteve, M. K. Oberthaler, D. J. Frantzeskakis, G. Theocharis, and P. G. Kevrekidis, Phys. Rev. Lett. 101, 130401 (2008).

[28] E. H. Lieb, Phys. Rev. 130, 1616 (1963).

[29] P. P. Kulish, S. V. Manakov, and L. D. Faddeev, Theor. Math. Phys. 28, 615 (1976).

[30] M. Ishikawa and H. Takayama, J. Phys. Soc. Jpn. 49, 1242 (1980).

[31] See Supplemental Material at http://link.aps.org/ supplemental/10.1103/PhysRevLett.111.020402 for the derivation of the effective Hamiltonian and for further results of the LL model in the weak coupling limit.

[32] V. Korepin, N. Bogoliubov, and A. Izergin, Quantum Inverse Scattering Method and Correlation Functions (Cambridge University Press, Cambridge, England, 1997).

[33] J. Sato, R. Kanamoto, E. Kaminishi, and T. Deguchi, arXiv:1204.3960.

[34] G. E. Astrakharchik and L. P. Pitaevskii, arXiv:1210.8337.

[35] M. Girardeau, J. Math. Phys. (Cambridge, Mass.) 1, 516 (1960).

[36] T. Tsuzuki, J. Low Temp. Phys. 4, 441 (1971).

[37] In fact, Fig. 4 strongly suggests that the convergence rate is $D^{-1}$.

[38] D. Draxler, J. Haegeman, T. J. Osborne, V. Stojevic, L. Vanderstraeten, and F. Verstraete (to be published). 